\title{
Targeting the PXR-TLR4 signaling pathway to reduce intestinal inflammation in an experimental model of necrotizing enterocolitis
}

\author{
Kezhen Huang ${ }^{1}$, Subhajit Mukherjee ${ }^{2}$, Vera DesMarais ${ }^{3}$, Joseph M Albanese ${ }^{4}$, Ektor Rafti ${ }^{1}$, Andrew Draghi II $^{1}$, \\ Leigh A Maher ${ }^{5}$, Kamal M Khanna ${ }^{6}$, Sridhar Mani ${ }^{2}$ and Adam P Matson ${ }^{1}$
}

BACKGROUND: There is substantial evidence that signaling through Toll-like receptor 4 (TLR4) contributes to the pathogenesis of necrotizing enterocolitis (NEC). Pregnane $X$ receptor (PXR), a xenobiotic sensor and signaling intermediate for certain hostbacterial metabolites, has been shown to negatively regulate TLR4 signaling. Here we investigated the relationship between PXR and TLR4 in the developing murine intestine and explored the capacity of PXR to modulate inflammatory pathways involved in experimental NEC.

METHODS: Wild-type and $\mathrm{PXR}^{-1-}$ mice were studied at various time points of development in an experimental model of NEC. In addition, we studied the ability of the secondary bile acid lithocholic acid (LCA), a known PXR agonist in liver, to activate intestinal PXR and reduce NEC-related intestinal inflammation.

RESULTS: We found a reciprocal relationship between the developmental expression of PXR and TLR4 in wild-type murine intestine, with PXR acting to reduce TLR4 expression by decreasing TLR4 mRNA stability. In addition, $\mathrm{PXR}^{-1-}$ mice exhibited a remarkably heightened severity of disease in experimental NEC. Moreover, LCA attenuated intestinal proinflammatory responses in the early stages of experimental NEC.

CONCLUSION: These findings provide proactive insights into the regulation of TLR4 in the developing intestine. Targeting PXR may be a novel approach for NEC prevention.

$\mathbf{N}$ ecrotizing enterocolitis (NEC) is the most common and lethal gastrointestinal emergency of premature infants, affecting 5-10\% of infants with a birth weight under $1,500 \mathrm{~g}$ (1). The mortality rate is between 20 and $30 \%$, and survivors are at significant risk for neurodevelopmental delays and lifelong impairment in gut function (2). The exact etiology of NEC remains unknown; however, several lines of evidence suggest an interaction between luminal bacteria and pattern recognition receptors in the intestinal epithelium. Studies in animal models and surgically resected preterm intestine suggest that signaling through Toll-like receptor 4 (TLR4), which recognizes lipopolysaccharide (LPS) of Gram-negative bacteria, is critical to NEC pathogenesis (3-5). Excessive stimulation of TLR4 leads to decreased epithelial proliferation, induction of apoptosis, and reduced barrier integrity (5-7), which may allow for bacteria to translocate across the epithelium culminating in the severe (uncontrolled) innate inflammatory response characteristic of the disease (1). Thus, identifying mechanisms of TLR4 control as they relate to the maintenance of intestinal barrier function could help in the design of NEC prevention strategies.

Pregnane X receptor (PXR), a nuclear receptor and xenobiotic sensor that is highly expressed in intestine and liver (8), has recently been identified as a negative regulator of TLR4 signaling $(9,10)$. PXR is activated by a broad range of endogenous and exogenous ligands, including steroids, bile acids, dietary compounds, and host-bacterial metabolites (8-11). In xenobiotic liver metabolism, PXR regulates the expression of genes encoding enzymatic pathways involved in detoxification and clearance, such as cytochrome P450s and multidrug resistance protein 1a (Mdrla) (P-glycoprotein) $(12,13)$. It is interesting to note that there is mutual suppression between xenobiotic metabolism and NF-KBmediated inflammation in the enterohepatic axis (14). Exposure to xenobiotic drugs that activate PXR can suppress inflammation (14); whereas, inflammation can reduce the expression of PXR-responsive genes (15). The ability of PXR to function as a signaling intermediate for certain hostbacterial metabolites and its opposing relationship to TLR4 and NF-KB activity has made it an attractive target for studying intestinal inflammatory disorders. As such, PXR has been identified as contributing to inflammatory bowel disease (IBD) in humans $(16,17)$ and animal models $(18)$. To date, however, there have been no studies investigating the role of PXR in regulating intestinal inflammation in NEC.

${ }^{1}$ Department of Pediatrics, University of Connecticut School of Medicine, Farmington, Connecticut; ${ }^{2}$ Department of Medicine and Genetics, Albert Einstein College of Medicine, Bronx, New York; ${ }^{3}$ Department of Anatomy and Structural Biology, Albert Einstein College of Medicine, Bronx, New York; ${ }^{4}$ Department of Pathology, Montefiore Medical Center, Bronx, New York; ${ }^{5}$ Department of Immunology, University of Connecticut School of Medicine, Farmington, Connecticut; ${ }^{6}$ Department of Microbiology, New York University Langone School of Medicine, New York, New York. Correspondence: Adam P. Matson (Amatson@UCHC.edu)

Received 7 July 2017; accepted 11 January 2018; advance online publication 21 February 2018. doi:10.1038/pr.2018.14 


\section{Articles | Huang et al.}

Although no experimental model of NEC fully recapitulates the clinical condition, many of the signaling pathways involved in NEC pathogenesis have been elucidated through the use of such systems (19). Indeed, the importance of TLR4 and its associated molecules in NEC pathophysiology has been demonstrated using neonatal mice subjected to a model of intermittent hypoxia and formula feeds, or variations thereof $(5-7,20)$. Thus, to better understand the pathogenesis of NEC relative to TLR4-dependent signaling, and explore the capacity of PXR to regulate NEC-associated inflammatory pathways, wild-type (WT) and $\mathrm{PXR}^{-1-}$ mice were studied at various time points of development and subjected to a wellestablished experimental model of NEC $(5-7,20,21)$. In addition, we studied the ability of the secondary bile acid lithocholic acid (LCA), a known PXR agonist in liver, to activate intestinal PXR and reduce NEC-associated intestinal inflammation. Secondary bile acids, such as LCA, are produced by luminal bacteria via the deconjugation of primary bile acids and are reported to have variable effects on the intestinal epithelium $(22,23)$. We speculated that when used at concentrations comparable to those that may be found in the neonatal intestine (24) and at levels sufficient to activate PXR in liver (11), LCA would activate intestinal PXR and reduce signaling via proinflammatory pathways thought to contribute to NEC. We observed a reciprocal relationship between the developmental expression of intestinal PXR and TLR4 and that $\mathrm{PXR}^{-1-}$ mice demonstrated a heightened inflammatory response during experimental NEC. Furthermore, activation of intestinal PXR through LCA reduced early proinflammatory responses that precede development of fullblown disease in the NEC model.

\section{METHODS}

\section{Reagents, Cell Culture, and Mice}

Ultrapure LPS (Escherichia coli 0111:B4) from Invivogen (San Diego, CA) was reconstituted in sterile water. LCA was from Sigma-Aldrich (St. Louis, MO) and reconstituted in methanol or directly dissolved in NEC formula. The rat small-intestinal cell line, IEC-6, was obtained from the American Type Culture Collection (ATCC, Manassas, VA) and maintained as per ATCC recommendations. Mouse primary intestinal organoids were isolated according to STEMCELL Technologies' (Cambridge, MA) mouse intestinal organoid isolation protocol and cultured in the mouse IntestiCult organoid growth medium (06005). C57BL/6 (WT) mice were from the Jackson Laboratory (Bar Harbor, ME). $\mathrm{PXR}^{-1-}$ mice (11) were generously provided by Dr. Sridhar Mani (Albert Einstein College of Medicine) and backcrossed into a C57BL/6 background. All animal procedures were approved by the University of Connecticut Health Animal Care and Use Committee and performed in accordance with the recommendations in the Guide for the Care and Use of Laboratory Animals of the National Institutes of Health.

\section{Quantitative Real-Time PCR}

Total RNA was extracted from either cultured IEC-6 enterocytes or ileal tissues using the RNeasy mini kit (Qiagen, Valencia, CA) and reverse transcribed ( $1 \mu \mathrm{g}$ of RNA) into cDNA using iScript reverse transcription supermix for RT-qPCR (Bio-Rad, Hercules, CA). The mRNAs for genes of interest were quantified by real-time PCR using the Bio-Rad CFX96 real-time system and primers obtained from Integrated DNA Technologies (Coralville, IA) (Predesigned qPCR
Assays). All results were normalized to the housekeeping gene $\beta$-actin.

\section{RNA Interference}

ON-TARGETplus rat Nr1i2 siRNA SMARTpool (L-099761-02) and ON-TARGETplus non-targeting siRNA (D-001810-01) were purchased from Dharmacon (Lafayette, CO). Small interfering RNA was transfected into IEC-6 cells for $48 \mathrm{~h}$ using Lipofectamine 2000 transfection reagent (ThermoFisher, Waltham, MA), followed by treatment as indicated.

\section{Induction of Experimental Mouse Necrotizing Enterocolitis}

Experimental NEC was induced in 7-day-old mice (WT or $\mathrm{PXR}^{-1-}$ ) using a protocol similar to that previously reported $(5-7,20,21)$. NEC formula (Similac (Abbott Nutrition, Columbus, OH) PM60/40 infant formula: Esbilac (PetAg, Hampshire, IL) canine milk replacer, 2:1) was supplemented with enteric bacteria made from a stock created from a stool specimen obtained from an infant with surgical NEC $(12.5 \mu \mathrm{l}$ stool slurry in $1 \mathrm{ml}$ formula) and fed by oral gavage five times per day ( $50 \mu \mathrm{l}$ per $\mathrm{g}$ mouse body weight). The mice also were exposed to hypoxia $\left(5 \% \mathrm{O}_{2}, 95 \% \mathrm{~N}_{2}\right)$ for 10 min twice daily for up to 4 days using a hypoxic chamber (Billups-Rothenberg, Del Mar, CA). Previous studies have demonstrated that this experimental NEC protocol induces intestinal inflammation and production of inflammatory cytokines that closely resembles human NEC $(5-7,20,21)$. Control mice remained with their mothers and were breast-fed ad libitum. After killing, terminal ileums were harvested $1 \mathrm{~cm}$ proximal to the ileocecal valve and fixed in $10 \%$ formaldehyde for histologic assessment or frozen in liquid nitrogen for quantitative real-time PCR. The severity of disease was determined on histologic sections stained with hematoxylin and eosin by a blinded investigator based on a previously published NEC scoring system from 0 (normal) to 3 (severe) (5). Immunohistochemistry was performed essentially as described (25). Formalin-fixed paraffin-embedded sections of mouse small intestine underwent antigen retrieval at pH 6 in Dako target retrieval solution (Dako, Carpinteria, CA) and incubated with a 1:200 dilution $(1 \mu \mathrm{g} / \mathrm{ml})$ of polyclonal rabbit antimouse PXR (Cloud Clone, Houston, TX). This primary antibody was detected with EnVision+ System-HRP anti-rabbit reagent (Dako) and visualized with a DAB substrate kit (Cell Marque, Rocklin, CA). PXR staining intensity was determined by scanning slides on the 3DHistec Pannoramic 250 Flash II slide scanner (SIG \#1S10OD019961-01) in the Analytical Imaging Facility at Albert Einstein College of Medicine.

\section{Isolation of Nuclei and Nuclear Run-On Assay}

These assays were performed according to the methods previously published (26) with minor modifications. Nuclei were isolated from cultured LS174T cells and immediately stored at $-80^{\circ} \mathrm{C}$ until analysis. For nuclear run-on assay, identical number of LS174T nuclei was used for preparation of nascent transcripts. To perform the nuclear run-on transcription, $5 \times 10^{6}$ nuclei were incubated in a reaction buffer ( $5 \mathrm{mM}$ Tris- $\mathrm{HCl}, \mathrm{pH} 8.0,2.5 \mathrm{mM} \mathrm{MgCl}_{2}, 150 \mathrm{mM}$ $\mathrm{KCl}, 1.0 \mathrm{mM}$ each of ATP, GTP, CTP) and $0.5 \mathrm{mM}$ biotin-16-UTP at $30{ }^{\circ} \mathrm{C}$ for $45 \mathrm{~min}$ in a final volume of $25 \mu \mathrm{l}$. The reaction was stopped by the addition of $60 \mathrm{U}$ RNase-free DNase and incubated further for $10 \mathrm{~min}$ at $37^{\circ} \mathrm{C}$. The nuclei were then lysed by the addition of lysis buffer containing $10 \mathrm{mM}$ Tris- $\mathrm{HCl}, 1 \%$ SDS, $5 \mathrm{mM}$ EDTA. The reaction mixtures were treated with $20 \mu \mathrm{l}$ of proteinase $\mathrm{K}(10 \mathrm{mg} / \mathrm{ml})$. RNA was extracted with TRIzol Reagent, ethanol-precipitated, and resuspended in $50 \mu \mathrm{l}$ RNase-free water. Biotinylated RNA was purified by adding streptavidin beads, followed by $2 \mathrm{~h}$ incubation at $25^{\circ} \mathrm{C}$ on a shaker. Beads were separated by centrifugation at $2,000 \mathrm{rpm}$ for $5 \mathrm{~min}$, and washed once with $2 \times$ SSC- $15 \%$ formamide for $10 \mathrm{~min}$ and twice with $2 \times$ SSC alone for $5 \mathrm{~min}$. Finally, beads were resuspended in $30 \mu \mathrm{l}$ DEPC-treated water. The biotinylated RNA solution was used for reverse-transcriptase cDNA synthesis and further real-time qPCR for the quantitative mRNA assay. 
Actinomycin D Chase

LS174T cells were plated into six-well tissue culture dishes and treated with rifampicin for $48 \mathrm{~h}$. After rifampicin treatment, $20 \mu \mathrm{g} / \mathrm{ml}$ of Actinomycin D, dissolved in DMSO was added in each well and RNA isolated at different time points starting at $t=0$ after actinomycin D exposure. RNA was subsequently used for reverse transcription and real-time qPCR analysis.

\section{Statistical Analysis}

Statistical analysis was performed using Prism 7 software (GraphPad, La Jolla, CA). Data are expressed in mean \pm SEM as indicated. Twotailed unpaired $t$-test was used for comparisons between two groups. Ordinary one-way analysis of variance (ANOVA) with Tukey's posttest was used when comparing fold changes between multiple groups, whereas Kruskal-Wallis one-way ANOVA with Dunn's post-test was used for multiple comparisons to a control group. Statistical significance was defined as a $P$ value $\leqslant 0.05$. The half-life of TLR4 mRNA was calculated according to the formula: $t_{1 / 2}=0.693 / \kappa$, where $\kappa=\ln (\mathrm{N} 0 / \mathrm{Nt}) \mathrm{t}$, with N0 being TLR 4 mRNA expression at $t=0$ and Nt being TLR4 mRNA expressions at various time points.

\section{RESULTS}

\section{Reciprocal Relationship Between PXR and TLR4 in the Developing Murine Intestine}

We first sought to characterize the relationship between expression of PXR and TLR4 in the developing intestine. To do so, ileal-jejunal tissues were harvested from prenatal (E16, E18) or postnatal (P1, P3, P7) WT mice, out to a time when mice are susceptible to experimental NEC (20,21), to determine PXR and TLR4 transcript levels by quantitative real-time PCR (qRT-PCR). As shown in Figure 1a, intestinal TLR4 expression was highest during the early prenatal period and decreased following delivery. In contrast, PXR expression was lowest during the early prenatal period and increased robustly around the time of delivery. PXR target genes (Mdr1a and Cyp3a11) demonstrated a similar pattern of expression (Figure 1bi,ii). These observations suggest a reciprocal relationship between the developmental expression of PXR and TLR4 in murine intestine.

To further explore this relationship, ileal-jejunal tissues were harvested from WT and $\mathrm{PXR}^{-1-}$ mice from E18 to postnatal day 7 and analyzed for TLR4 expression. There were no differences in intestinal TLR4 expression between $\mathrm{PXR}^{-/-}$ and WT mice at any of the time points evaluated (Figure 1c). Thus, despite the reciprocal relationship between expression of PXR and TLR4 in WT mice, the absence of PXR did not significantly impact baseline TLR4 expression during the prenatal or early postnatal periods.

\section{PXR Negatively Regulates the TLR4 Pathway by Decreasing TLR4 mRNA Stability}

Given the observed inverse relationship between expression of PXR and TLR4 in the developing intestine, we next sought to better define the mechanism(s) by which PXR regulates the TLR4 pathway. To determine whether PXR regulates TLR4 at the transcriptional level, we performed nuclear run-on analysis using human colon epithelial LS174T cells treated with the human PXR agonist rifampicin (27). As shown in Figure 2a, upon activation of PXR with rifampicin, we observed a steady increase in MDR-1 gene transcription but
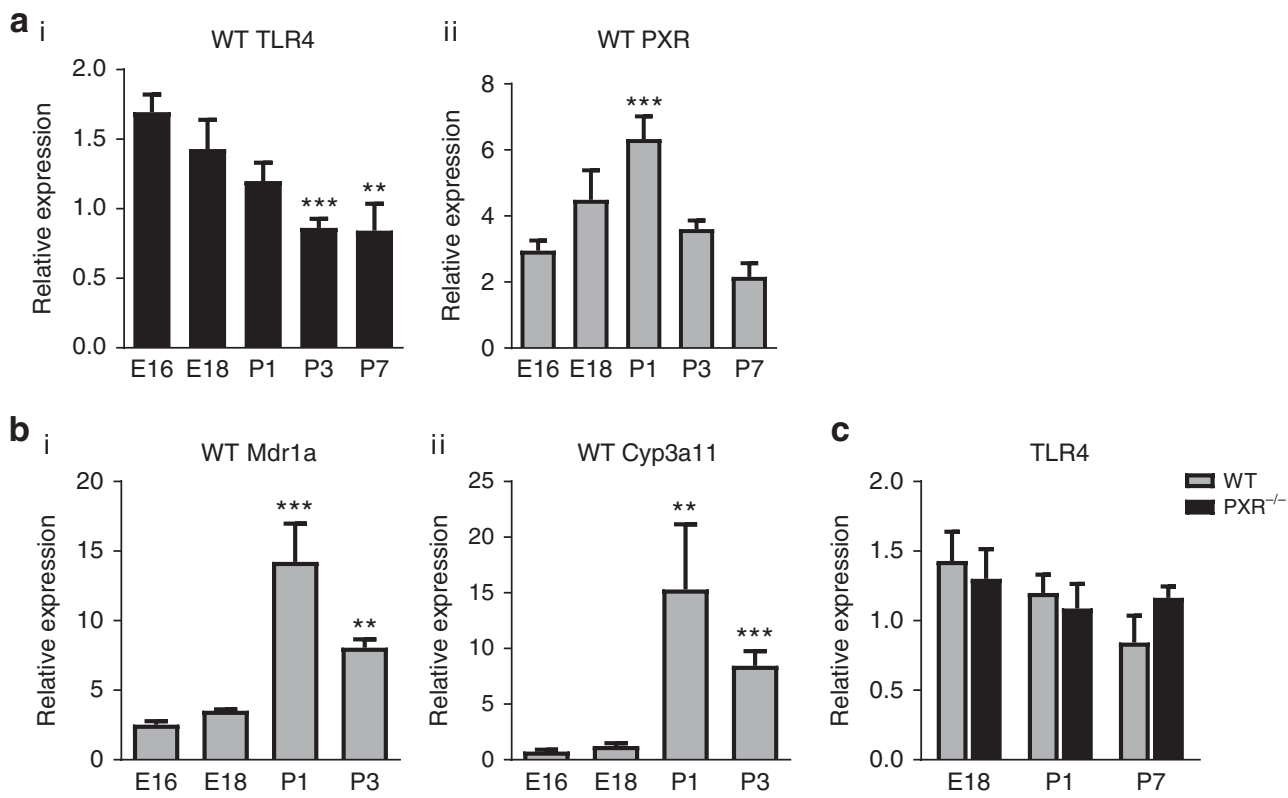

Figure 1. Ontogeny of intestinal PXR and TLR4 expression. (a) qRT-PCR showing mRNA expression levels of TLR4 (i) and PXR (ii) relative to $\beta$-actin in the intestines of C57BL/6 (WT) mice at the indicated prenatal (E) or postnatal (P) times. Shown are the means and SEMs from three separate experiments with $6-11$ mice per group. ${ }^{* *} P \leqslant 0.01,{ }^{* * *} P \leqslant 0.001$ for TLR4 or PXR levels vs. E16 mice. (b) Expression levels of PXR target genes in intestinal tissues of WT mice. ${ }^{* *} P \leqslant 0.01,{ }^{* * *} P \leqslant 0.001$ for levels of Mdr1a (i) or Cyp3a11 (ii) vs. E16 mice. Results are from two separate experiments with 7-8 mice per group. Comparisons in a and b were by Kruskal-Wallis one-way analysis of variance (ANOVA) with Dunn's post-test. (c) Expression levels of TLR4 mRNA in intestinal tissues of WT and $\mathrm{PXR}^{-1-}$ mice. There were no differences in levels of TLR4 transcripts between the groups at the indicated times by unpaired $t$ test. Results are representative of four separate experiments with 4-8 mice per group. PXR, pregnane $X$ receptor; qRT-PCR, quantitative real-time PCR; TLR4, Toll-like receptor 4. 

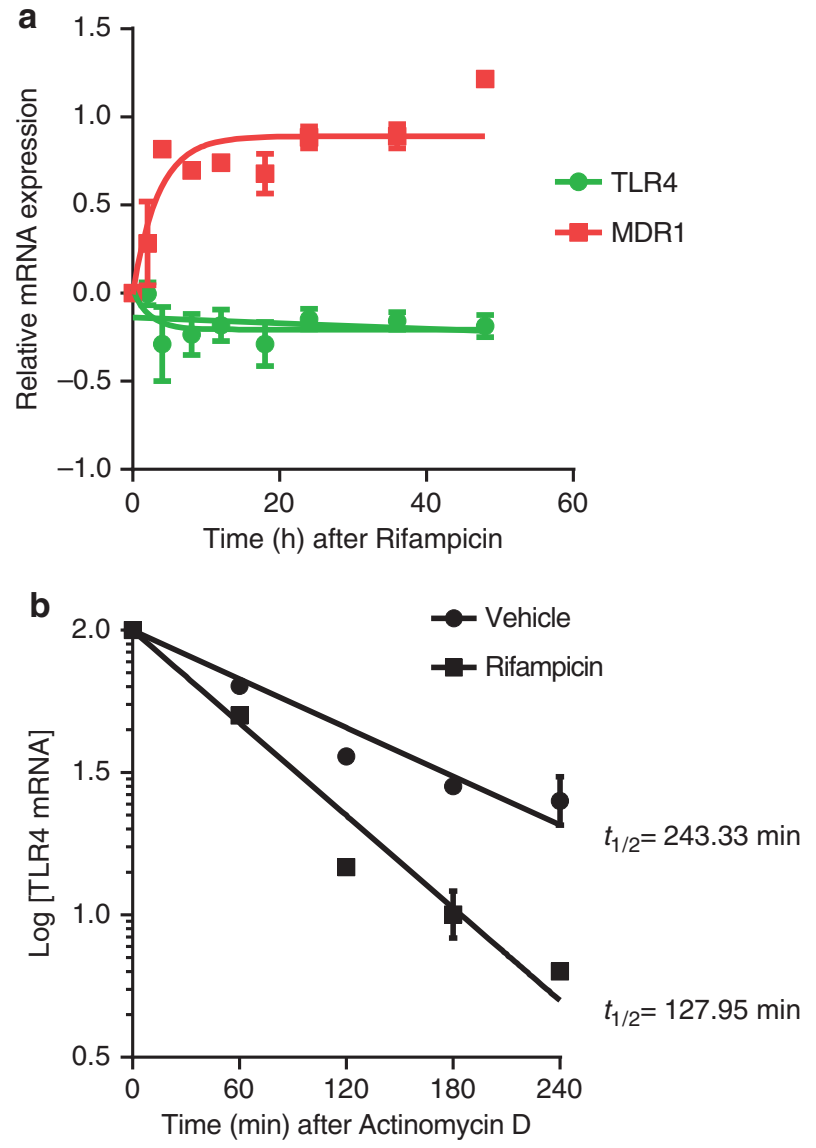

Figure 2. PXR-mediated regulation of TLR4 signaling is not directly dependent on TLR4 gene transcription but instead through regulating TLR4 mRNA stability. (a) Nuclei of LS174T cells were isolated at various time points after treatment with the human PXR ligand rifampicin $(10 \mu \mathrm{M})$ and used in the nuclear run-on transcription reaction. After the reaction, biotinylated RNA was purified and used for cDNA synthesis and real-time qPCR. The transcription efficiency of TLR4 and classical PXR target gene, MDR-1, were analyzed relative to that of $\beta$-actin. (b) Actinomycin D $(20 \mu \mathrm{g} / \mathrm{ml})$ chase experiment was performed in LS174T cells treated with or without rifampicin to evaluate TLR4 mRNA stability. After rifampicin treatment for $48 \mathrm{~h}$, TLR4 mRNA half-life decreased from $\sim 243$ to $\sim 127 \mathrm{~min}$. PXR, pregnane $\mathrm{X}$ receptor; TLR4, Toll-like receptor 4.

no change in TLR4 transcription (Figure 2a). We then considered the possibility that PXR acts post-transcriptionally to attenuate expression of TLR4. To this end, we conducted Actinomycin D chase experiments and found that rifampicin treatment decreased the half-life of TLR 4 mRNA by 47\% compared to untreated cells (Figure 2b). Together, these observations show that the effect of PXR activation by rifampicin on TLR4 gene expression in not a direct transcriptional event, but instead it is due to a PXRmediated post-transcriptional effect on TLR4 mRNA stability.

WT Mice Demonstrate Reduced PXR Protein Expression and PXR-Deficient Mice Demonstrate a Heightened Inflammatory Response During Experimental NEC

It is known that there is mutual repression between the TLR4 and PXR signaling pathways $(14,15)$. Given the critical involvement of TLR4 signaling to NEC pathogenesis, we next sought to determine whether exposure to NEC-associated intestinal inflammation would alter PXR expression and whether the absence of PXR would increase susceptibility to NEC. Experimental NEC was induced in WT and $\mathrm{PXR}^{-1-}$ pups using a well-characterized model that involves gavage feeding with formula and exposure to twice-daily hypoxia $(5-7,20,21)$. In this model, WT mice demonstrate severe intestinal inflammation by day 4; thus we killed some mice at earlier time points to clarify the inflammatory processes that underlie the development of full-blown disease. We first examined intestinal tissues from WT control (breastfed) and WT NEC mice that were immunostained for PXR. As shown in Figure 3a, at an early stage (day 2) of the NEC protocol, WT control mice demonstrated PXR-positive stain intensity that was almost two-fold higher than that found in WT NEC mice $(31.52 \%$ vs. $17.27 \%)$.

We next compared WT NEC mice to $\mathrm{PXR}^{-1-}$ NEC mice at a similar early stage of the NEC protocol. As shown in Figure $\mathbf{3 b}-\mathbf{d}$, by gross inspection and histopathological assessment, the gut injury was more severe in the $\mathrm{PXR}^{-1-}$ mice. Experimental NEC also induced a profound increase in transcripts for the proinflammatory cytokine IL-6, which was $\sim 10$-fold greater in intestinal tissues obtained from $\mathrm{PXR}^{-/-}$ mice as compared to WT mice. Exposure to the NEC protocol also resulted in a significant increase in TLR4 expression in WT mice, which was markedly exacerbated in the absence of PXR (Figure 3ei,ii). These observations indicate that intestinal proinflammatory cascades progress faster in the absence of PXR and further suggest, consistent with other studies $(9,10,14)$, that PXR suppresses TLR4 signaling in NEC-associated inflammation. Additionally, markers of intestinal barrier function were more notably disrupted in $\mathrm{PXR}^{-1-}$ mice. Experimental NEC resulted in reduced levels of MUC2, a predominant secretory mucin produced by goblet cells, in $\mathrm{PXR}^{-1-}$ mice as compared to WT mice (Figure 3eiii). At baseline, connexin-43 (Cx43), the major connexin involved in maintaining gap-junctional intercellular communication, was lower in $\mathrm{PXR}^{-1-}$ control mice vs. WT controls, but then dramatically increased in $\mathrm{PXR}^{-1-}$ mice subjected to experimental NEC (Figure 3eiv), which could indicate gapjunction disassembly with accumulation of dysfunctional isoforms (28).

\section{LCA Activates Intestinal PXR and Suppresses LPS-Induced Proinflammatory Cytokine Expression In Vitro}

To study the physiological function of intestinal PXR in vitro, we treated IEC- 6 enterocytes with the secondary bile acid LCA, a known PXR agonist in liver (11). (Figure 4ai,ii) shows that LCA stimulated the transcription of PXR and Mdrla, a major gene target of PXR, in a dose-dependent manner, indicating that LCA treatment promotes activation of PXR in intestinal epithelial cells. Evidence of developing toxicity was observed when LCA concentrations exceeded $>200 \mu \mathrm{M}$, thus for subsequent in vitro experiments, LCA was used at concentrations below this threshold. To examine the potential 


\section{Targeting PXR-TLR4 in experimental NEC $\quad$ Articles}

a
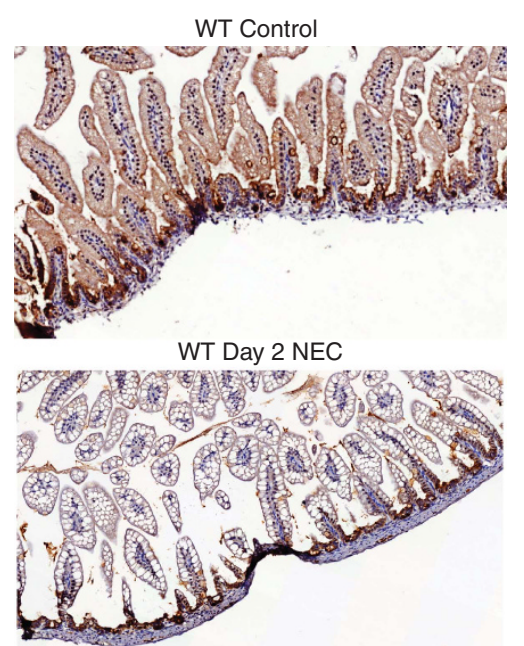

b
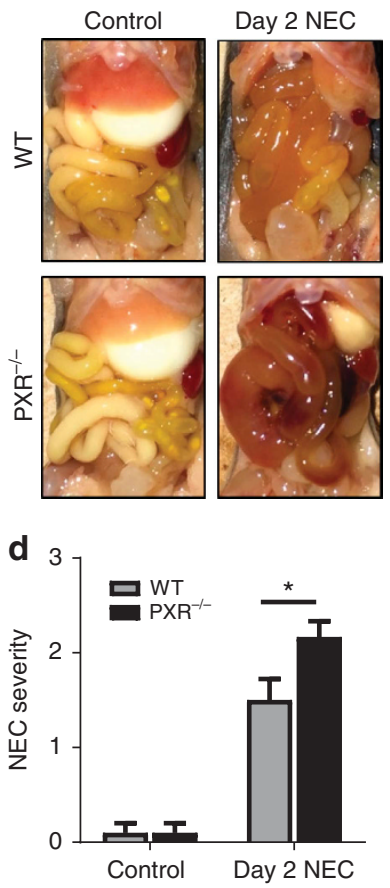

C
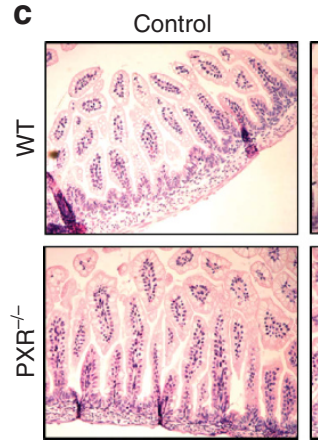

$\mathbf{e}_{\mathrm{i}}$

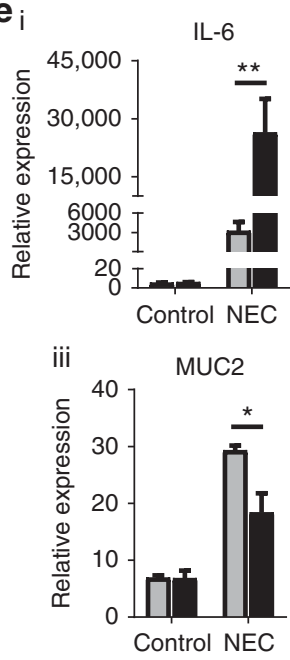

Day 2 NEC

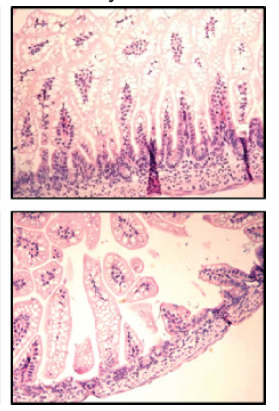

ii $\quad$ TLR4

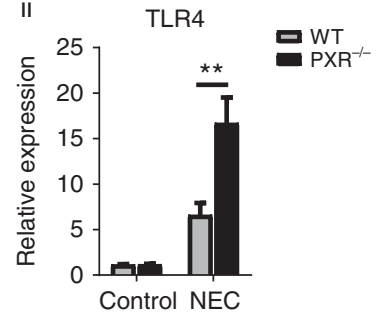

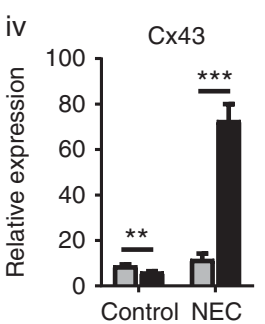

Figure 3. WT mice demonstrate reduced PXR expression and $\mathrm{PXR}^{-/-}$mice demonstrate a heightened inflammatory response following induction of experimental NEC. (a) Intestinal tissues from WT control (breastfed) and WT NEC mice (day 2 of the NEC protocol) immunostained for PXR. (b) Gross appearance of the intestine immediately after killing of WT or PXR ${ }^{-/-}$mice that were controls or subjected to the NEC protocol (day 2). (c) H\&Estained sections of the terminal ileum in the indicated groups. (d) Quantification of NEC severity in WT or PXR ${ }^{-1-}$ mice. ${ }^{*} P<0.05$ when compared between the groups by unpaired $t$ test. Results are representative of three separate experiments with 3 (for each control group) to 6 (for each group at day 2 NEC) mice per group. (e) qRT-PCR showing the levels of transcripts for IL-6 (i), TLR4 (ii), MUC2 (iii), and Cx43 (iv) relative to those for $\beta$-actin in intestinal tissues collected from WT or PXR ${ }^{-/-}$mice that were controls or subjected to experimental NEC. ${ }^{*} P \leqslant 0.05,{ }^{* *} P \leqslant 0.01,{ }^{* * *} P \leqslant 0.001$ when compared between groups by unpaired $t$-test. Results are representative of three separate experiments with at least three mice per group. H\&E, hematoxylin and eosin; NEC, necrotizing enterocolitis; PXR, pregnane X receptor; qRT-PCR, quantitative real-time PCR; TLR4, Toll-like receptor 4.

of PXR to suppress TLR4 signaling cascades, we pretreated IEC-6 cells with LCA prior to the stimulation with LPS. (Figure 4bi,ii) shows that LCA pretreatment reduced the LPS-induced transcription of both IL- 6 and TNF $\alpha$ by $\sim 50 \%$ $(P<0.05$ and $P<0.01$, respectively).

\section{LCA Suppresses LPS-Induced IL-6 Transcription in a PXR- \\ Dependent Manner}

It is known that LCA can activate intestinal receptors other than PXR, such as the vitamin D receptor (29). Therefore, we next sought direct evidence that the ability of LCA to suppress LPS-induced proinflammatory responses in intestinal tissues was PXR-dependent. To do so, we knocked down PXR in IEC-6 enterocytes using PXR-specific siRNA. Following the knockdown, IEC-6 cells exposed to LCA demonstrated a three-fold reduction in PXR mRNA as compared to those treated with scrambled siRNA (data not shown). Under conditions of reduced PXR expression, LCA pretreatment no longer suppressed LPS-induced transcription of IL-6; instead, an enhancement in the levels of IL-6 transcripts was observed (Figure 5ai). In contrast, LCA still suppressed the LPS-induced transcription of TNF $\alpha$ (Figure 5aii), suggesting that IL-6 and TNF $\alpha$ are differentially regulated downstream of TLR4 signaling cascades. In this series of experiments, the induction of proinflammatory transcripts following stimulation of IEC-6 cells with LPS was less pronounced than in Figure 4 b; however, the results were suggestive that LCA may diminish the LPSinduced transcription of IL-6 in intestinal tissues via PXR.

To further investigate this possibility, we isolated primary intestinal organoids (30) from WT and $\mathrm{PXR}^{-1-}$ mice, and exposed them to LPS following pretreatment or not with LCA. As shown in Figure 5bi, LCA pretreatment of WT intestinal organoids reduced LPS-induced transcription of IL- 6 by over $90 \%$. In contrast, LCA pretreatment did not alter LPS-induced transcription of IL-6 in intestinal organoids generated from $\mathrm{PXR}^{-1-}$ mice (Figure 5bii). This result strongly suggests that LCA suppression of LPS-induced IL-6 expression in intestinal tissues is PXR-dependent. LCA did 

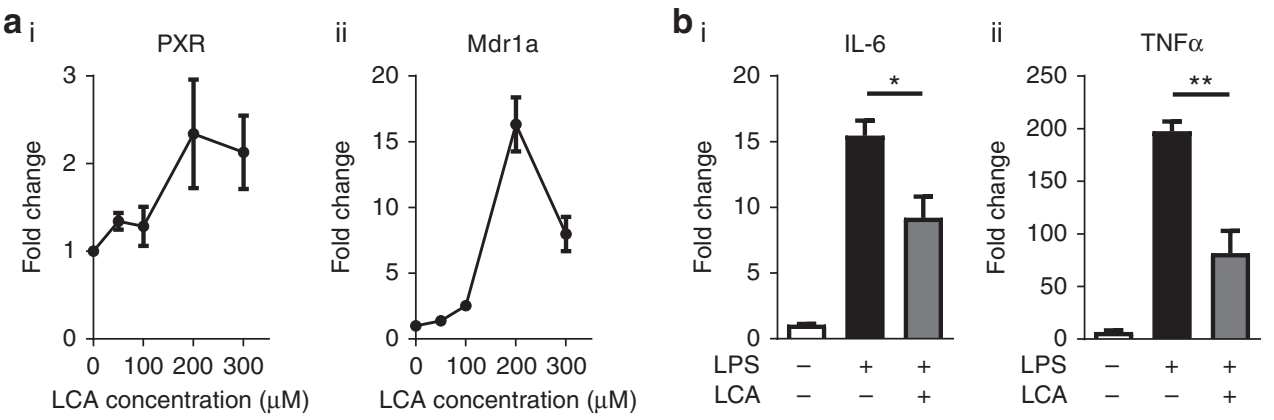

Figure 4. The PXR agonist and secondary bile acid LCA attenuates LPS-induced cytokine production in IEC-6 enterocytes. qRT-PCR showing (a) dose response of LCA treatment on IEC- 6 enterocytes for the induction of PXR (i) and the PXR-responsive gene Mdr1a (ii). (b) Expression levels of transcripts for IL-6 (i) and TNFa (ii) in IEC-6 enterocytes treated with $100 \mu \mathrm{M}$ of LCA followed by $2 \mathrm{~h}$ stimulation with LPS (1 $\mu \mathrm{g} / \mathrm{ml})$. Results shown are for IEC- 6 cells in triplicate. Fold changes were calculated relative to untreated cells and normalized to $\beta$-actin. ${ }^{*} P \leqslant 0.05,{ }^{* *} P \leqslant 0.01$ by unpaired $t$ test. LCA, lithocholic acid; LPS, lipopolysaccharide; PXR, pregnane X receptor; qRT-PCR, quantitative real-time PCR.
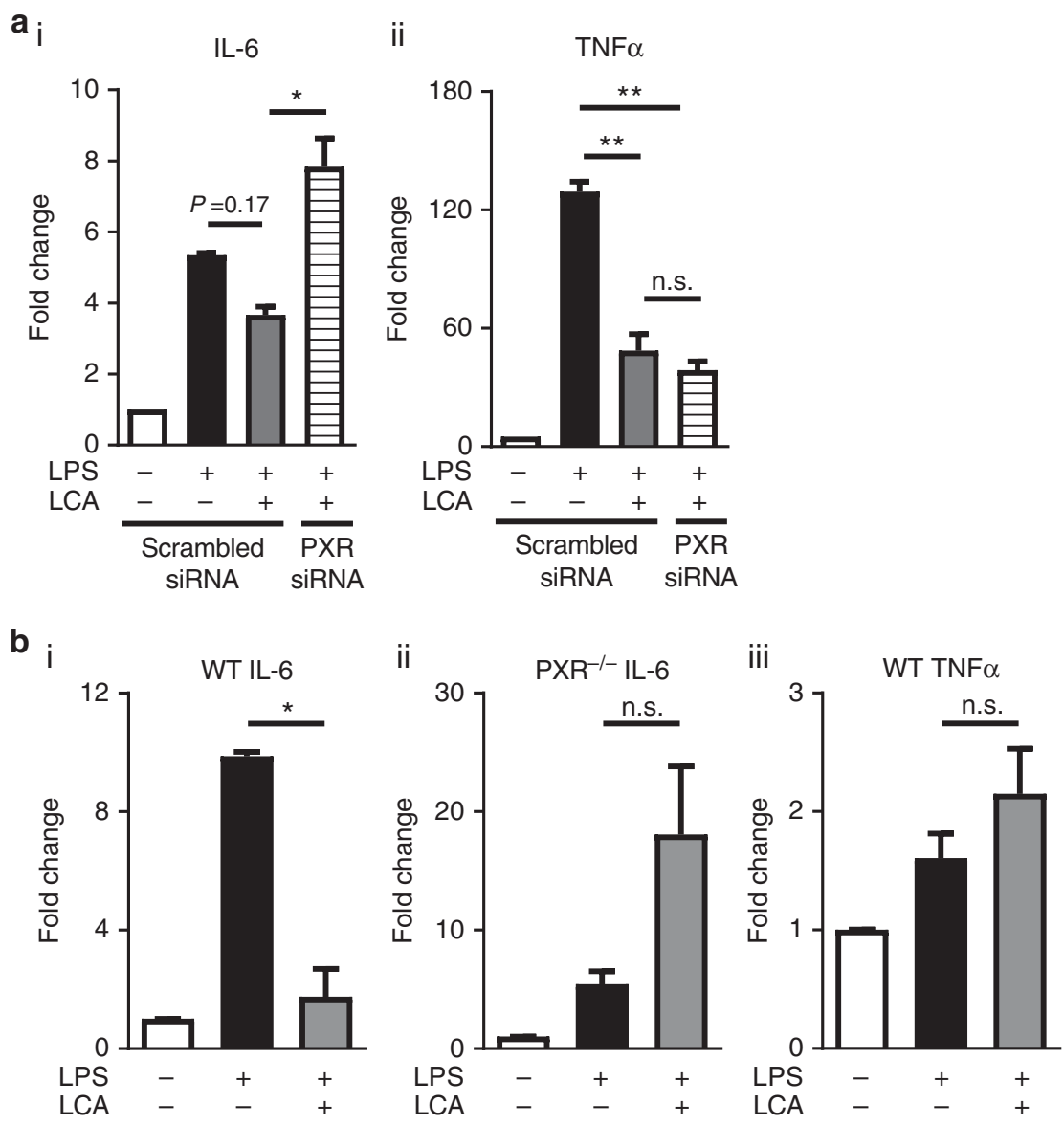

Figure 5. The ability of LCA to diminish LPS-induced production of IL-6 in intestinal tissues is PXR-dependent. (a) Expression levels of LPS-induced transcripts for IL-6 (i) and TNFa (ii) in IEC-6 enterocytes pretreated or not with $100 \mu \mathrm{M}$ of LCA (24 h) in the absence or presence of PXR siRNA or scrambled siRNA $(200 \mu \mathrm{M})$. (b) Expression levels of LPS-induced transcripts for IL-6 (i and ii) and TNF-a (iii) in intestinal organoids obtained from WT or PXR ${ }^{-/-}$mice that were pretreated or not with $100 \mu \mathrm{M}$ of LCA (16 h). Exposures to LPS were for $2 \mathrm{~h}$ at $1 \mu \mathrm{g} / \mathrm{ml}(\mathbf{a})$ or $4 \mathrm{~h}$ at $10 \mu \mathrm{g} / \mathrm{ml}(\mathbf{b})$. Results shown are for experiments performed in triplicate. Fold changes were calculated by comparing to untreated cells or organoids and normalized to $\beta$-actin. ${ }^{*} P \leqslant 0.05,{ }^{* *} P \leqslant 0.01$, by ANOVA with Tukey's multiple comparison test (a), or unpaired $t$-test (b); ANOVA, analysis of variance; LCA, lithocholic acid; LPS, lipopolysaccharide; n.s., not significant; PXR, pregnane $X$ receptor.

not alter LPS-induced transcription of TNF $\alpha$ in WT intestinal organoids (Figure 5biii), providing additional evidence that transcription of this cytokine is regulated downstream of
TLR4 signaling in a PXR-independent manner and potentially by an LCA receptor that is present in rat IEC-6 cells but absent in murine intestinal organoids. 
Targeting PXR-TLR4 in experimental NEC | Articles
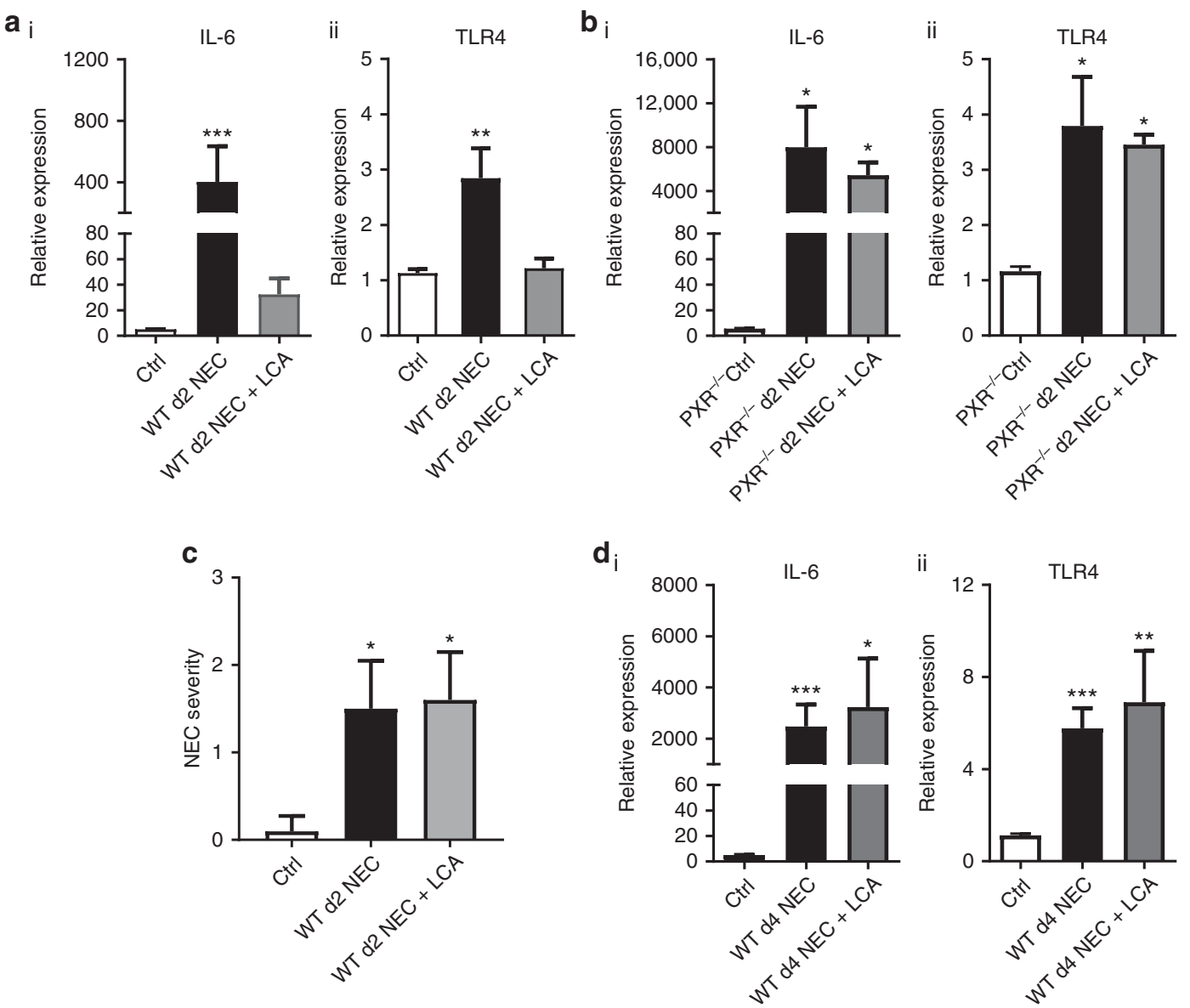

Figure 6. LCA reduces intestinal inflammation in the early stages of NEC in a PXR-dependent manner. (a) Expression levels of transcripts for IL-6 (i) and TLR4 (ii) in intestinal tissues obtained from WT controls (Ctrl) or WT mice subjected to early stage (day 2) of NEC +/ - LCA supplementation. (b) Expression levels of transcripts for (i) IL-6 and (ii) TLR4 in intestinal tissues obtained from PXR ${ }^{-/-}$Ctrl or PXR ${ }^{-1-}$ mice subjected to early stage NEC +/- LCA supplementation. (c) Quantification of NEC severity in WT mice subjected to early stage NEC +/ - LCA supplementation. (d) Expression levels of transcripts for (i) IL-6 and (ii) TLR4 in intestinal tissues obtained from WT Ctrl or WT mice subjected to day 4 NEC +/- LCA supplementation. Expression levels were relative to $\beta$-actin. ${ }^{*} P \leqslant 0.05,{ }^{* *} P \leqslant 0.01,{ }^{* * *} P \leqslant 0.001$ when compared to Ctrl group by Kruskal-Wallis ANOVA with Dunn's posttest. Results are representative of two to four separate experiments with three (controls) and five to six mice (day 2 NEC) per group in C, and at least four mice per group in (a, b, and $\mathbf{d})$. ANOVA, analysis of variance; LCA, lithocholic acid; LPS, lipopolysaccharide; NEC, necrotizing enterocolitis; PXR, pregnane $\mathrm{X}$ receptor.

LCA Reduces Intestinal Transcription of IL-6 and TLR4 at an Early Stage of Experimental NEC in a PXR-Dependent Manner In the next series of experiments, we sought to determine whether LCA could protect against NEC-associated inflammation in a PXR-dependent manner. WT and $\mathrm{PXR}^{-/-}$ mice were subjected to the NEC protocol described previously $(5-7,20,21)$; however, in this series, formula mixtures administered by oral gavage were supplemented or not with $0.5 \%$ LCA. At an early stage (day 2 ) following initiation of the NEC protocol, the transcription of IL-6 and TLR4 in the terminal ileums of WT mice receiving LCA was reduced compared to WT mice not given LCA, indicating that LCA protected against the upregulation of these proinflammatory markers (Figure 6ai,ii). In contrast, LCA failed to reduce these proinflammatory markers in the terminal ileums of $\mathrm{PXR}^{-1-}$ mice (Figure 6bi,ii). This observation suggests that during early stages of NEC-associated inflammation, LCAmediated reduction in IL-6 and TLR4 is PXR-dependent.
Despite these reductions, there was no difference in histologic grading of NEC severity (Figure 6c). Furthermore, as NEC progressed to late stage (day 4), the levels of transcripts for IL-6 and TLR4 were comparable between LCA and non-LCA fed WT mice (Figure 6di,ii).

\section{DISCUSSION}

Excessive stimulation of TLR4 within the intestinal epithelium is speculated to serve as a prequel to NEC by initiating signaling cascades that result in intestinal barrier breakdown (5-7). Subsequently, bacterial translocation and propagation of the profound inflammatory response to infiltrating bacteria leads to intestinal necrosis and the systemic manifestations that are characteristic of this disease (1). Inhibition of TLR4 signaling in the intestinal epithelium has been shown to enhance enterocyte proliferation and limit enterocyte apoptosis $(6,21,31)$, and could therefore be utilized as a potential route for NEC prevention (21). In this study, we evaluated the 


\section{Articles | Huang et al.}

capacity of PXR, a xenobiotic sensor and negative regulator of TLR4 signaling $(9,10)$, to limit NEC-associated inflammatory pathways. We show that a reciprocal relationship exists between the developmental expression of intestinal PXR and TLR4, PXR protein levels are reduced in WT mice subjected to experimental NEC, and that PXR-deficient mice exhibit a remarkably heightened inflammatory response following exposure to the NEC protocol. Moreover, we provide evidence that the secondary bile acid and commensal byproduct, LCA, reduces $\mathrm{NEC}$-associated intestinal inflammatory responses in a PXR-dependent manner. Taken together, these findings suggest that targeting intestinal PXR may be a novel approach to reduce intestinal injury in conditions, such as NEC, that are attributed to excessive TLR4 stimulation. It is known that following ligand binding, PXR heterodimerizes with retinoid $\mathrm{X}$ receptor (RXR) to form a PXR/RXR complex, which directly interacts with the p65 subunit of NF- $\mathrm{BB}$ thereby limiting TLR4 signaling cascades $(8,32)$. Here we provide evidence that at least one additional mechanism for PXR to limit NEC-related inflammatory responses is via the regulation of TLR4 mRNA stability.

In both humans (4) and mice (33), intestinal expression of TLR4 is higher early in gestation compared to later time points, which suggests a developing role for TLR4 in epithelial differentiation. The possibility exists that elevated expression of TLR4 in the preterm intestine is the normal state and that pathology arises from a combination of premature delivery and postnatal microbial colonization. Nevertheless, only $\sim 5-$ $10 \%$ of the most susceptible infants develop NEC (1), which suggests that protective mechanisms exist to suppress the deleterious effects of TLR4 activation. Our data support the notion that PXR is a central regulator of TLR4-mediated control of intestinal barrier function (9) and expand the spectrum of intestinal disorders in which PXR may play a protective role to include NEC (9,16-18). In WT intestinal tissues, the robust increase in PXR transcripts, and PXR-target genes Mdr1a and Cyp3a11, noted shortly after delivery coincided with a reduction in TLR4. Our discovery that PXR regulates TLR4 mRNA stability further suggests that accumulation of xenobiotic PXR agonists in the postnatal intestine may serve as a switch to limit TLR4 expression after birth. Additionally, in the absence of PXR, mice subjected to NEC had a marked increase in TLR4 expression compared to WT mice and demonstrated increased susceptibility to intestinal injury.

Prior to the induction of experimental NEC, we compared baseline levels of transcripts for proteins associated with intestinal inflammation or barrier homeostasis in $\mathrm{PXR}^{-/-}$vs. WT mice, as an indicator of their vulnerability to intestinal injury during the perinatal period. We observed no elevation in baseline levels of TLR4 transcripts in prenatal or early postnatal $\mathrm{PXR}^{-1-}$ mice compared to age-matched WT controls. This is in contrast to a recent report that utilized adult $\mathrm{PXR}^{-1-}$ mice, in which the absence of PXR was associated with increased baseline enterocyte TLR4 expression (9). It is possible that insufficient levels of commensal microbiota were present during the perinatal period to induce TLR4 expression over WT mice. Additional mechanisms of early postnatal intestinal TLR4 control, such as epidermal growth factor (EGF) ingested with the breast milk may also be involved (21). Nevertheless, because our model includes gavage feeding with enteric bacteria isolated from a preterm infant with NEC, the observed increase in TLR4 mRNA following initiation of the NEC protocol strongly suggests that microbiota-derived signals play a major role in regulating TLR4 expression, particularly in the setting of reduced PXR activity.

The possibility that intestinal dysbiosis contributes to NEC susceptibility is supported by several studies demonstrating that the fecal microbiome of infants that develop NEC is dominated by LPS-containing Proteobacteria $(34,35)$. Alternatively, an abundance of strict anaerobes is negatively associated with NEC, which suggests that these bacteria and/or their products may trigger protective mechanisms (35). In our study, we used the secondary bile acid and known liver PXR agonist, LCA, to evaluate its ability to dampen NEC-associated intestinal inflammation. LCA is produced from the conversion of primary bile acids by commensal bacteria possessing $7 \alpha$-dehydroxylase activity (36); of the various bacteria inhabiting the human intestine, several strains of Clostridium $(36,37)$ and Bacteroides (38) possess $7 \alpha$-dehydroxylase activity. Both ex vivo experiments and analysis of intestinal tissues obtained from early NEC show that LCA diminishes intestinal proinflammatory responses in a PXR-dependent manner. Conversely, LCA did not reduce histologic severity or alter intestinal proinflammatory responses at later time points. This likely reflects the nature of the model, which involves repeated, combined insults to the intestine, surpassing LCA's protective effects. Alternatively, the reduction in PXR expression following initiation of the NEC protocol may account for decreased responsiveness to LCA. It is conceivable that providing higher doses of LCA would result in greater levels of protection; however, high doses of LCA are also associated with liver toxicity (11). In future studies, additional PXR ligands with greater activating potential or lower levels of systemic toxicity should be evaluated for their capacity to protect against NEC. It was recently demonstrated that tryptophan metabolites (e.g., Indole 3-propionic acid) produced by intestinal commensals regulate intestinal barrier function through $\operatorname{PXR}(9,39)$. The combined influence of microbial small molecule output on NEC susceptibility is an intriguing area of investigation.

Interestingly, Cx43 mRNA was significantly reduced in $\mathrm{PXR}^{-1-}$ mice as compared to WT controls, but then demonstrated a profound increase upon the induction of experimental NEC. Cx43 is the most abundant connexin regulating gap junction communication between enterocytes, and protein levels are reported to be reduced in experimental NEC and patients with IBD (40). It is known that Mdr1adeficient mice, which develop spontaneous colitis, have accumulation of differentially phosphorylated isoforms of Cx43 representing a marker of gap junction disruption during 


\section{Targeting PXR-TLR4 in experimental NEC $\quad$ Articles}

intestinal injury (28). Thus, under conditions of cellular stress, the absence of PXR, which regulates Mdrla activity, may also result in the accumulation of dysfunctional isoforms of $\mathrm{Cx} 43$. This possibility remains to be determined but could account for the large increase in Cx43 transcripts measured by RTPCR. There were no differences in baseline levels of IL- 6 or MUC2 transcripts between $\mathrm{PXR}^{-1-}$ and WT mice; however, their pattern of expression following the induction of NEC were consistent with greater levels of intestinal damage in the $\mathrm{PXR}^{-1-}$ mice.

Despite the established association between reduced PXR expression and IBD $(16,17)$, there have been no studies evaluating the contribution of PXR in human NEC. It is interesting to note that while PXR agonists can protect against DSS-induced colitis, PXR-deficient mice do not show increased susceptibility to this model of distal colonic injury (18). In contrast, in animal models, small intestinal disease appears to be enhanced in the absence of PXR $(9,14)$. This suggests that the large intestine, as compared to the small intestine, may have compensatory mechanisms in place that can be activated in the absence of PXR (18). NEC involves the small bowel and ileocecal junction more often than the large bowel, supporting the notion that PXR may play a regulatory role in NEC pathophysiology at these anatomic sites. Furthermore, compensatory mechanisms may not be fully established in the preterm infant, in which case the protective effect of PXR signaling may be more critical in the large bowel.

In summary, we have now expanded the role of PXR as a central regulator of TLR4 signaling to include suppression of inflammatory pathways in the early phase of experimental NEC. Further studies will determine if PXR plays a similar regulatory role in human NEC and explore the potential utility of microbiome-, pharmacologic- or dietary-related PXR agonists as novel approaches for preventing this devastating condition of prematurity.

\section{ACKNOWLEDGMENTS}

We gratefully acknowledge David Hackam and his laboratory staff, Johns Hopkins Children's Center, for their advice in performing the NEC model; and Justin Radolf, University of Connecticut School of Medicine, for his mentorship and review of the manuscript.

\section{STATEMENT OF FINANCIAL SUPPORT:}

This work was supported by funds made available through a Connecticut Children's Medical Center Young Investigator Award (A.P.M.), NCl P30CA013330 (Analytical Imaging Facility at the Albert Einstein College of Medicine, V.D., S.M.), SIG\#1S10OD019961-01 (Analytical Imaging Facility, V.D.), Broad Medical Research Program (\#362520; S.M.), R01 CA161879 (S.M.) and Department of Defense Grant\# PR160167 (K.M. K., S.M.).

\section{DISCLOSURE:}

US patent application \# PCT/US2013/072709 filed by Albert Einstein College of Medicine on behalf of S.M. and S.M. (https://www.google.com/ patents/WO2014088982A1?cl=en).
Disclosure: The authors declare no competing financial interests.

\section{REFERENCES}

1. Neu J, Walker WA. Necrotizing enterocolitis. N Engl J Med 2011;364: 255-64.

2. Fitzgibbons SC, Ching $\mathrm{Y}, \mathrm{Yu}$ D, et al. Mortality of necrotizing enterocolitis expressed by birth weight categories. J Pediatr Surg 2009;44: 1072-5.

3. Jilling T, Simon D, Lu J, et al. The roles of bacteria and TLR4 in rat and murine models of necrotizing enterocolitis. J Immunol 2006;177:3273-82.

4. Nanthakumar N, Meng D, Goldstein AM, et al. The mechanism of excessive intestinal inflammation in necrotizing enterocolitis: an immature innate immune response. PLoS ONE 2011;6:e17776.

5. Leaphart CL, Cavallo J, Gribar SC, et al. A critical role for TLR4 in the pathogenesis of necrotizing enterocolitis by modulating intestinal injury and repair. J Immunol 2007;179:4808-20.

6. Sodhi CP, Shi XH, Richardson WM, et al. Toll-like receptor-4 inhibits enterocyte proliferation via impaired beta-catenin signaling in necrotizing enterocolitis. Gastroenterology 2010;138:185-96.

7. Neal MD, Sodhi CP, Jia H, et al. Toll-like receptor 4 is expressed on intestinal stem cells and regulates their proliferation and apoptosis via the p53 up-regulated modulator of apoptosis. J Biol Chem 2012;287:37296-308.

8. Kliewer SA, Moore JT, Wade L, et al. An orphan nuclear receptor activated by pregnanes defines a novel steroid signaling pathway. Cell 1998;92:73-82.

9. Venkatesh M, Mukherjee S, Wang $\mathrm{H}$, et al. Symbiotic bacterial metabolites regulate gastrointestinal barrier function via the xenobiotic sensor PXR and Toll-like receptor 4. Immunity 2014;41:296-310.

10. Qiu Z, Cervantes JL, Cicek BB, et al. Pregnane X receptor regulates pathogen-induced inflammation and host defense against an intracellular bacterial infection through Toll-like receptor 4. Sci Rep 2016;6:31936.

11. Staudinger JL, Goodwin B, Jones SA, et al. The nuclear receptor PXR is a lithocholic acid sensor that protects against liver toxicity. Proc Natl Acad Sci USA 2001;98:3369-74.

12. Bertilsson G, Heidrich J, Svensson K, et al. Identification of a human nuclear receptor defines a new signaling pathway for CYP3A induction. Proc Natl Acad Sci USA 1998;95:12208-13.

13. Sakaeda T, Nakamura T, Okumura K. MDR1 genotype-related pharmacokinetics and pharmacodynamics. Biol Pharm Bull 2002;25: 1391-400.

14. Zhou C, Tabb MM, Nelson EL, et al. Mutual repression between steroid and xenobiotic receptor and NF-kappaB signaling pathways links xenobiotic metabolism and inflammation. J Clin Invest 2006;116: 2280-9.

15. Aitken AE, Richardson TA, Morgan ET. Regulation of drug-metabolizing enzymes and transporters in inflammation. Annu Rev Pharmacol Toxicol 2006;46:123-49.

16. Langmann T, Moehle C, Mauerer R, et al. Loss of detoxification in inflammatory bowel disease: dysregulation of pregnane $\mathrm{X}$ receptor target genes. Gastroenterology 2004;127:26-40.

17. Dring MM, Goulding CA, Trimble VI, et al. The pregnane X receptor locus is associated with susceptibility to inflammatory bowel disease. Gastroenterology 2006;130:341-8.

18. Shah YM, Ma X, Morimura K, Kim I, Gonzalez FJ. Pregnane X receptor activation ameliorates DSS-induced inflammatory bowel disease via inhibition of NF-kappaB target gene expression. Am J Physiol Gastrointest Liver Physiol 2007;292:G1114-22.

19. Lin J, Hackam DJ. Worms, flies and four-legged friends: the applicability of biological models to the understanding of intestinal inflammatory diseases. Dis Model Mech 2011;4:447-56.

20. Sodhi CP, Neal MD, Siggers R, et al. Intestinal epithelial Toll-like receptor 4 regulates goblet cell development and is required for necrotizing enterocolitis in mice. Gastroenterology 2012;143:708-18.

21. Good M, Sodhi CP, Egan CE, et al. Breast milk protects against the development of necrotizing enterocolitis through inhibition of Toll-like receptor 4 in the intestinal epithelium via activation of the epidermal growth factor receptor. Mucosal Immunol 2015;8:1166-79. 


\section{Articles | Huang et al.}

22. Ward JB, Lajczak NK, Kelly OB, et al. Ursodeoxycholic acid and lithocholic acid exert anti-inflammatory actions in the colon. Am J Physiol Gastrointest Liver Physiol 2017;312:G550-8.

23. Milovic V, Teller IC, Faust D, Caspary WF, Stein J. Effects of deoxycholate on human colon cancer cells: apoptosis or proliferation. Eur J Clin Invest 2002;32:29-34.

24. Murphy GM, Signer E. Bile acid metabolism in infants and children. Gut 1974;15:151-63.

25. Wang $\mathrm{H}$, Venkatesh $\mathrm{M}, \mathrm{Li} \mathrm{H}$, et al. Pregnane $\mathrm{X}$ receptor activation induces FGF19-dependent tumor aggressiveness in humans and mice. J Clin Invest 2011;121:3220-32.

26. Zhang MX, Ou H, Shen $\mathrm{YH}$, et al. Regulation of endothelial nitric oxide synthase by small RNA. Proc Natl Acad Sci USA 2005;102:16967-72.

27. Watkins RE, Wisely GB, Moore LB, et al. The human nuclear xenobiotic receptor PXR: structural determinants of directed promiscuity. Science 2001;292:2329-33.

28. Ey B, Eyking A, Klepak M, et al. Loss of TLR2 worsens spontaneous colitis in MDR1A deficiency through commensally induced pyroptosis. J Immunol 2013;190:5676-88.

29. Ikura T, Ito N. Crystal structure of the vitamin D receptor ligand-binding domain with lithocholic acids. Vitam Horm 2016;100:117-36.

30. Wilson SS, Tocchi A, Holly MK, Parks WC, Smith JG. A small intestinal organoid model of non-invasive enteric pathogen-epithelial cell interactions. Mucosal Immunol 2015;8:352-61.

31. Neal MD, Sodhi CP, Dyer M, et al. A critical role for TLR4 induction of autophagy in the regulation of enterocyte migration and the pathogenesis of necrotizing enterocolitis. J Immunol 2013;190:3541-51.
32. Gu X, Ke S, Liu D, et al. Role of NF-kappaB in regulation of PXR-mediated gene expression: a mechanism for the suppression of cytochrome P-450 3A4 by proinflammatory agents. J Biol Chem 2006;281:17882-9.

33. Gribar SC, Sodhi CP, Richardson WM, et al. Reciprocal expression and signaling of TLR4 and TLR9 in the pathogenesis and treatment of necrotizing enterocolitis. J Immunol 2009;182:636-46.

34. Mai V, Young CM, Ukhanova $\mathrm{M}$, et al. Fecal microbiota in premature infants prior to necrotizing enterocolitis. PLoS ONE 2011;6:e20647.

35. Warner BB, Deych E, Zhou Y, et al. Gut bacteria dysbiosis and necrotising enterocolitis in very low birthweight infants: a prospective case-control study. Lancet 2016;387:1928-36.

36. Studer N, Desharnais L, Beutler M, et al. Functional intestinal bile acid 7alpha-dehydroxylation by Clostridium scindens associated with protection from Clostridium difficile infection in a gnotobiotic mouse model. Front Cell Infect Microbiol 2016;6:191.

37. Kitahara M, Sakamoto M, Benno Y. PCR detection method of Clostridium scindens and C. hiranonis in human fecal samples. Microbiol Immunol 2001;45:263-6.

38. Hirano S, Masuda N. Enhancement of the 7 alpha-dehydroxylase activity of a gram-positive intestinal anaerobe by Bacteroides and its significance in the 7-dehydroxylation of ursodeoxycholic acid. J Lipid Res 1982;23:1152-8.

39. Dodd D, Spitzer MH, Van TW, et al. A gut bacterial pathway metabolizes aromatic amino acids into nine circulating metabolites. Nature 2017;551: 648-52.

40. Leaphart CL, Qureshi F, Cetin S, et al. Interferon-gamma inhibits intestinal restitution by preventing gap junction communication between enterocytes. Gastroenterology 2007;132:2395-411. 